\title{
TWINE: Supporting Assessment of Trustworthiness of Web Information Using Linked Data
}

\author{
Jarutas Pattanaphanchai \\ ICT, Faculty of Science \\ Prince of Songkla University \\ Hat Yai, Songkhla, 90112, Thailand \\ Email: jarutas.p@psu.ac.th
}

\author{
Kieron O'Hara \\ Electronics and Computer Science \\ University of Southampton, Southampton \\ SO17 1BJ, United Kingdom \\ Email: kmo@ecs.soton.ac.uk
}

\author{
Wendy Hall \\ Electronics and Computer Science \\ University of Southampton, Southampton \\ SO17 1BJ, United Kingdom \\ Email:wh@ecs.soton.ac.uk
}

\begin{abstract}
Trustworthiness of information is an important factor to be considered when users consume data on the Web environment, because it affects the decision of the users whether or not to utilize the information they find. In the worst case, by consuming untrustworthy information, users can arrive at a wrong decision. Therefore, it is necessary for Web users to have tool that can help them to assess the trustworthiness of the information they are consuming. In this paper, we proposed a prototype for helping Web users to evaluate the trustworthiness of Web information, by providing them supportive data about the Web information they are considering. Our prototype collects and integrates useful metadata based on practice trustworthiness criteria, using Linked Data, and presents the supportive information to the users. The results showed that using our prototype can improve the user's ability to assess the information, and whether or not to trust information. The users' trust level of the information was increased. In addition, the users were satisfied with the provided supportive information for helping them evaluate the trustworthiness of Web information.
\end{abstract}

\section{INTRODUCTION}

The World Wide Web is a massive source of information which allows anyone to publish anything. However, it lacks in terms of quality control over the publishing process. Accordingly, the trustworthiness of published information is concerned, since consuming untrustworthy information may lead to incorrect or unfavourable decisions. Therefore, Web users require critical criteria and tools to help them evaluating the information they are consuming. A study in Web credibility and information quality proposed trustworthiness criteria for assessing the credibility of Web information. Users can evaluate information based on criteria such as the identity of the author (e.g. name, position, title), the expertise of the author, or the date of publication in order to support their judgements. Moreover, the supportive information can increase the Web users' ability to assess the information and help them to determine whether the information they have found is trustworthy [1]-[4]. However, in today's Web, the relevant data might not be available to gather, or it is difficult to collect or integrate from several sources in order to build supportive information for supporting the evaluation of the trustworthiness of Web information.

Linked data is an approach for publishing and connecting structured data on the Web, in order to construct the data on the Web in machine-readable form and providing the feature of linking data between different sources. Using Linked data, the meaning of information content is explicitly defined, and can be linked to- or from- other external data sets. Linked data uses Resource Description Framework (RDF) to define typed statements, which may refer to any object (tangible or abstract) in the world [5]. As a result, Linked data provides a generic publishing method, which makes it easier to discover and integrate data from a large scale of data sources [6].

In this paper, we proposed a tool with the properties of collecting, integrating, and presenting useful supportive information, based on rational practical criteria using Linked data to support user's Web trustworthiness evaluation process. This paper is organised as follows: section II, we discuss related work. In section III, we present our trustworthiness of Web information prototype. Then, we discuss the evaluation process of the prototype in section IV and the analysis and results of the evaluation in section V. Finally, we draw the paper to a close by summarising our work in section VI.

\section{RELATED WORK}

Trustworthiness of Web information is a challenging issue in the Web environment. There are two areas of study that proposed a solution to assess the quality or credibility of information on the Web, namely information quality and Web credibility.

Information quality research area proposed a set of criteria or methods, that can be used to assess the quality of Web data and its data sources [4], [7]-[9]. Bizer and his colleagues proposed the WIQA - Information Quality Assessment Framework - which is a set of software components that can be used by applications for processing information of an uncertain quality. In addition, the researchers presented an application, called the WIQA browser, which employed their proposed framework to display the framework implementation in a real-world scenario. The WIQA browser extracts structure information on the web pages it visits; then stores this information together with provenance meta-information as a set of Named Graphs. Then, the browser uses filtering and an explanation engine to filter stored information and to generate explanations about filter decisions, which are then presented to the users [10]. Similarly, Ramachandran and his colleagues proposed a trustworthy and high-quality information retrieval system. The system provided an enhanced web search engine which provides the trustworthiness of search results. It used five factors (provenance, authority, age, popularity, and related links) to calculate the trustworthiness of Web information [11]. 
Web credibility focused on factors that affected to the credibility of information, which considers the individual Web site as the source of information. Therefore, the assessment of credibility in this context tries to evaluate the credentials of the Web site in question. There have been a number of studies on the issue of Web site credibility with proposed criteria that affect the credibility of Web information. Such as design appearance, information focus, accuracy of information, name recognition and reputation, advertising, bias of information, affiliations, the author's credentials or expertise and currency [3], [12]. Miyamori and his colleagues presented a system, called WISDOM, which is an information credibility analysis based on natural language processing. The WISDOM system needs to collect web pages using a spider, which it then stores in its local storage. Then, the stored web pages are analysed and classified based on content, sender, appearance, and social valuation criteria. The results show a list of related Web pages, which were classified by content, sender, concept, or opinion [13]. Atrey and his colleagues proposed a method for dynamically computing the trust level of Web sites of different domains based on the semantic similarity. The proposed approach considered the level of trust of Web sites by comparing the similarity of that Web site with the defined trusted Web sited, which was defined by the user survey [14].

Our work differs from previous discussed work in that our prototype automatically collects useful information, without requiring to go to a certain page for extracting useful metadata, and integrates collected metadata using Linked data. In addition, our prototype present the collected supportive information to the user to support their evaluation process. Our prototype aims to train users to be more critical in evaluating the trustworthiness of Web information by providing them with basic information; and by also providing them with additional supportive information to help them assess the information. In the next section, we will discuss the details of the construction of our prototype.

\section{TRUSTWORTHINESS OF WEB INFORMATION EVAluation APPLiCATION PROTOTYPE}

we present a prototype tool, called Twine, with properties of gathering and integrating useful metadata using Linked data in order to create and present supportive information to users for supporting their judgments of the trustworthiness of Web information. The prototype tool is implemented based on our trustworthiness of Web information evaluation framework [15]. As our case study, we chose to build a prototype to search for academic publications. The details of prototype architecture, a data model, and a prototype are discussed in the next section.

\section{A. TWINE System Architecture}

The system architecture of our prototype is illustrated in Fig.1. The prototype consists of four main functions; namely, input, generating an HTML page, metadata integration, and output functions. Moreover, as part of the metadata integration function, an integrated metadata graph is generated. In turn, this metadata graph is used to create JSON [16] data to display the results using the output function. The detail of each function is discussed in the next section.

- Input Function: the input function receives the search terms from the users and it calls the Google API to search for information based on the search terms. In this research, we focus on academic publications as a case study. In particular, we select the publications which are stored on a research repository using the ePrints (the repositories of electronic copies of research literature [17]).

- HTML Page Generation Function: this function generates the html page which is used as the interface between the users and the prototype. It does this from a page layout template, which is written using the Mako language (a template library provides a non-XML syntax that can be compiled into Python modules).

- Metadata Integration Function: the function consists of two main components; namely, trustworthiness criteria and metadata creation. The trustworthiness criteria component provides the criteria and indicators in which the metadata generation function should gather data based on them in order to create metadata graph. Based on the indicators of the trustworthiness criteria [18], the metadata creation module starts with building the basic metadata graph which describes the basic information of publications such as the title, the date, and the types of publication. These metadata are gathered from the page itself. Then, the metadata creation module retrieves further metadata of each publication by querying these additional metadata from the ePrints RDF data store using the publications' URIs and authors' URIs. The collected metadata are aggregated in order to build a metadata graph based on the data model discussed in the next section. This metadata graph is used in the output function to create the output to be displayed to the users.

- Output Function: The output function uses the metadata graph from the metadata integration function to create the results to display to the users. We use a TriQL.P query [19] to query the metadata from the metadata graph based on the authority criterion which explicitly indicates the quality of published data from the publisher. The equation for calculating the suggested trustworthiness score of Web information is the score from the authority criterion and the sum of the product of the usefulness score of the indicators in three criteria with the weighting value of these suggested indicators [15]. Therefore, the suggested trustworthiness score of the $i^{t h}$ result is given by

$$
T_{i}=S_{A, i}+\sum_{d \in D} U_{d} \cdot W_{d} \cdot P_{i, d}
$$

where

$$
P_{i, d}= \begin{cases}1, & \text { if } \mathrm{d} \text { is matched in result } \mathrm{i} \\ 0, & \text { otherwise }\end{cases}
$$

and where D is the set of indicators in accuracy, currency, and relevance criteria, $\mathrm{U}_{\mathrm{d}}$ is the usefulness score of indicators, $\mathrm{d}$, and $\mathrm{W}_{\mathrm{d}}$ is the weighting value of indicators, $\mathrm{d}$

Further to the above equations, $S_{A, i}$ is the score representing the combined authority of the authors of the paper, i. In this research, we take importance 


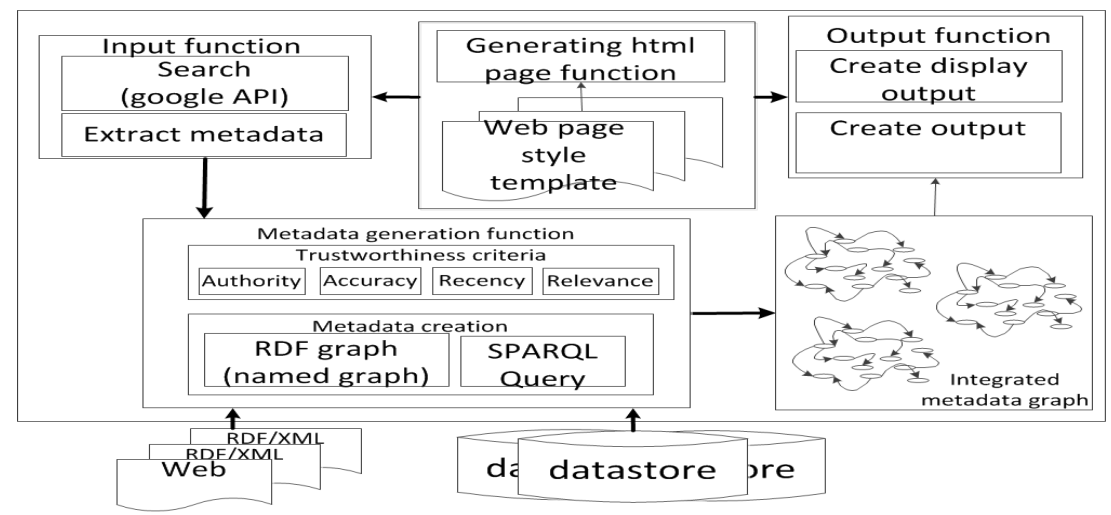

Fig. 1: The system architecture of the Twine prototype

of and broad impact of authors on the research area into account because the expertise and good reputation of authors in the community can indicate the quality of information they produced. We considered the expertise of authors by considering how often they are cited using the h-index [20]. However, an issue with the h-index is that it is unbounded. Therefore, we need to bound the effect of the h-index on the score in order to control the effect of the h-index, which might dominate the score in the authority criterion. As a result, the score of author in the author list is computed based on the sum of the individual author scores multiplied by the usefulness score and weighting value of the indicators in authority criterion and the bounded $\mathrm{h}$-index value of each author. The equation to calculate the authority score for the set of authors of paper $i$, $A_{i}$, is given by

$S_{A, i}=\frac{1}{\left|A_{i}\right|} \sum_{a \in A_{i}}\left[1-\left(\frac{1}{1+h_{a}}\right)+\sum_{k \in K} U_{k} \cdot W_{k} \cdot P_{k, a}\right]$

where

$P_{k, a}= \begin{cases}1, & \text { if indicator } \mathrm{k} \text { matches for author a } \\ 0, & \text { otherwise }\end{cases}$

where $h_{a}$ is h-index of author $a, A_{i}$ is the set of authors of result $\mathrm{i}, \mathrm{K}$ is the set of indicators in authority criteria, $\mathrm{U}_{\mathrm{k}}$ is the usefulness score of indicators, $\mathrm{k}$, and $\mathrm{W}_{\mathrm{k}}$ is the weighting value of indicators, $\mathrm{k}$.

The suggested trustworthiness score and the other supportive information are stored and made available in JSON format, which then are interpreted and shown to the users in a way that is easy to understand. The results of the prototype are displayed in order of decreasing suggested trustworthiness score. Furthermore, the other supportive information are shown as a combination of textual and visual elements such as time scales.

\section{B. TWINE Data Model}

We employ the named graphs data model [21] to represent our metadata as a metadata graph. Moreover, we use the Semantic Web Publishing Vocabulary (SWP) [22] to express the basic provenance information of the gathered metadata. In this prototype, the provenance information states the authorising relationship between a named graph and an authority in the form of a warrant. The authorizing relationship indicates a commitment between the authority and the content of the graph, and represents the properties by stating by whom the gathered metadata is asserted or quoted or the information's validity. We use the TriX syntax [23] to describe our named graph. When the prototype gathers metadata from the RDF links which are returned from search results or when it queries additional metadata from RDF data stores, the prototype creates a new named graph for each search result. It extracts provenance information from the RDF documents from the search results' RDF links and attaches that information to the metadata graph.

Fig.2 illustrates a data model which is used in our prototype. A graph which is built from this data model is called the metadata graph. Within the context of Twine, a metadata graph presents about the academic publications which are built in the form of graphs. A data model consists of three types of named graphs:

- Named graph of search results: This describes all the publications from the search results for which the user is interested in assessing the trustworthiness. It describes each search result by its publication URI.

- Named graph of publication metadata: This describes the information regarding the publications; namely, the authors of the publication (one author or multiple authors), the date that it was published, the title of the publication, the status of the publication, the type of publication, its abstract, and the provenance information of it authority.

- Named graph of metadata about the author: This represents the authors' credentials and expertise such as their list of publications or projects, their qualifications or the URL of their homepage.

\section{TWINE Application Prototype}

We designed our interface to be as simple as possible which has a text field that users can fill their search terms in order to retrieve the information they want. In addition to basic search results, the prototype included the necessary supportive information to help them evaluate the trustworthiness of Web 


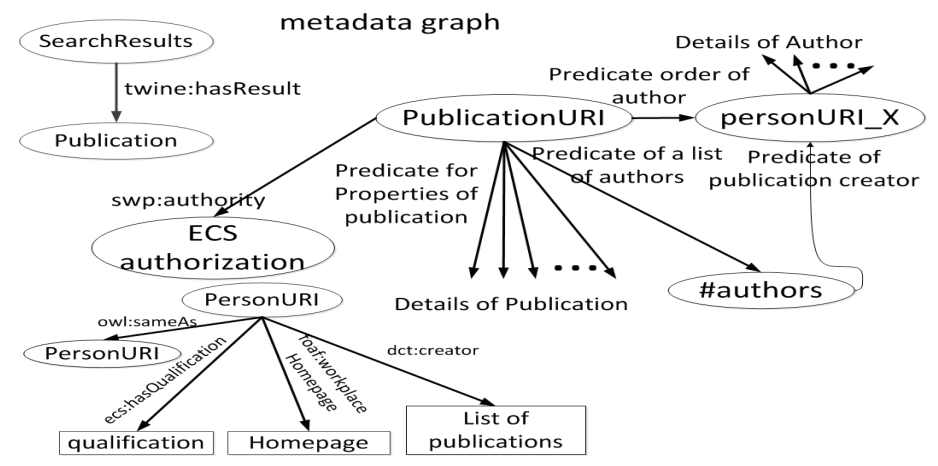

Fig. 2: A data model of the Twine prototype.

information. Moreover, our prototype have options for users to specify the area in which they are interested and how many pieces of information they can manage in one page.

\section{EVAluation of TWINE PROTOTYPE}

We set hypothesis as using a tool which provides supportive information that is gathered and integrated the supportive information using Linked data helps users to more critically evaluate the trustworthiness of Web information they are looking for. More specifically, the users increase their trust level in their decision whether or not to trust information they have found. We focused on the academic publications as a case study.

In order to test our hypotheses, we constructed a repeatedmeasures study in which the same participants take part in two experimental conditions. This study was approved by University of Southampton ethics committee (ethics reference number: 6800). We designed to evaluate the Twine prototype using two types of Twine tool environment:

- A control Twine prototype, called Twine1, which only provides basic information about the search results such as the title of the publication, the authors' names, and the number of times that the publication has been referenced.

- An experimental Twine prototype, called Twine2, which provides the basic information available in the control prototype, but also provides additional supportive information which is more detailed about the publications and authors; namely, the details of authors (e.g. position, workplace, qualifications, number of publications, projects, etc.), the editorial process, and the status of the publications. In addition, the prototype provided the explanation for why this publication would be trustworthy for the users.

We divided our experimental into four sessions. We used a priori power analysis to define the number of participants taking part in each session of the study. Given the effect size as 1.0 (large effect), the $\alpha$-level as 0.10 , and the statistical power as 0.8 , the minimum sample size of participants we needed to recruit for each session was estimated as eight participants. Therefore, in total, we needed 32 participants

We recruited 32 participants who are postgraduate students (who are just starting their studies, or who have been studying for no more than 2.5 years). We aimed to investigate the difference between users' decisions how much they trust information they have found depending on whether they are given just the basic information or provided with additional supportive information. Each group of participants was asked to search for publications relating to the topic of either privacy or semantic web using both Twine tool environments described above in a different order. In addition, they were asked to rate the trustworthiness score of the top ten search results in the provided questionnaire. After the participants completed all the tasks, we asked them to rate their level of satisfaction and influential of the supportive information provided to help them to assess the trustworthiness of Web information. In the next section, we discuss an analysis of the data from the study and the results.

\section{ANALysis AND Results OF THE STUdy}

This study investigated whether there were any significant differences in a user's decisions whether or not to trust the Web information between when they were provided with basic information about the Web information and when they were provided with additional supportive information. We investigated whether using supportive information which was gathered and integrated using Linked Data allowed the user to improve their ability to evaluate the trustworthiness of Web information. We analysed the results from the participants' answers with regards to our hypothesis as discussed in section IV.

\section{A. The trust level of the user in the Web information}

In the study, the participants were asked to rate their level of trust of information displayed by the search results using a Likert scale.The scale used in this question has seven points. They are very untrustworthy (1), mostly untrustworthy (2), somewhat untrustworthy (3), neither untrustworthy or trustworthy (4),somewhat trustworthy (5), mostly trustworthy (6), very trustworthy (7). Then, we calculated the average of the trust level from the top ten search results of each participant. We used a paired-samples t-test in order to investigate the difference between the trust levels of users when given basic information about the Web information (Twine1) and when given additional supportive information (Twine2). Results from the t-test data analysis showed that there was a statistically significant difference in the trust level of the users. On average, 
TABLE I: A paired samples test of the trustworthiness score between control and experiment prototypes

\begin{tabular}{|c|c|c|c|c|c|c|c|c|c|}
\hline & \multicolumn{5}{|c|}{ Paired Differences } & \multirow{3}{*}{$\mathrm{t}$} & \multirow{3}{*}{ df } & \multirow{3}{*}{$\begin{array}{l}\text { Sig.(2- } \\
\text { tailed) }\end{array}$} \\
\hline & & \multirow[t]{2}{*}{ Mean } & \multirow{2}{*}{$\begin{array}{l}\text { Std. } \\
\text { Devi- } \\
\text { ation }\end{array}$} & \multirow{2}{*}{$\begin{array}{c} \\
\text { Std. } \\
\text { Error } \\
\text { Mean } \\
\end{array}$} & \multicolumn{2}{|c|}{$\begin{array}{l}95 \% \text { Confidence } \\
\text { Interval } \\
\text { of the Difference }\end{array}$} & & & \\
\hline & & & & & Lower & Upper & & & \\
\hline Pair 1 & $\begin{array}{l}\text { Trust score with basic information } \\
\text { Trust score with additional information }\end{array}$ & $-0.62 \%$ & $0.67 \%$ & $0.08 \%$ & $-0.78 \%$ & $-0.45 \%$ & -7.39 & 63 & 0.001 \\
\hline
\end{tabular}

TABLE II: A one-sample t-test of the satisfaction level of participants

\begin{tabular}{|l|l|l|l|l|l|l|}
\hline \multirow{2}{*}{} & \multicolumn{6}{|c|}{ Test value $=1$ (not at all satisfied) } \\
\cline { 2 - 7 } & \multirow{2}{*}{} & $\mathrm{t}$ & $\mathrm{df}$ & Sig. (2-tailed) & $\begin{array}{c}\text { Mean } \\
\text { Difference }\end{array}$ & $\begin{array}{c}\text { 95\% Confidence Interval } \\
\text { of the Difference }\end{array}$ \\
\cline { 5 - 8 } & & & & Lower & Upper \\
\hline Satisfaction score & 21.947 & 31 & 0.001 & 2.688 & 2.44 & 2.94 \\
\hline
\end{tabular}

TABLE III: One-sample t-test of the influence level of the supportive information on the participants' judgments

\begin{tabular}{|c|c|c|c|c|c|c|}
\hline & \multicolumn{6}{|c|}{ Test value $=1$ (not at all influential) } \\
\hline & \multirow[t]{2}{*}{$\mathrm{t}$} & \multirow[t]{2}{*}{$\mathrm{df}$} & \multirow[t]{2}{*}{ Sig. (2-tailed) } & \multirow{2}{*}{$\begin{array}{c}\text { Mean } \\
\text { Difference }\end{array}$} & \multicolumn{2}{|c|}{$\begin{array}{l}95 \% \text { Confidence Interval } \\
\text { of the Difference }\end{array}$} \\
\hline & & & & & Lower & Upper \\
\hline Influence of provided supportive data & 22.512 & 31 & 0.001 & 2.719 & 2.47 & 2.97 \\
\hline
\end{tabular}

TABLE IV: One-sample test for level of agreement over the helpfulness of the explanation

\begin{tabular}{|l|l|l|l|l|l|l|}
\hline & \multicolumn{5}{|c|}{ Test value = 3 (Neither agree nor disagree) } \\
\cline { 2 - 8 } & \multirow{2}{*}{} & $\mathrm{t}$ & $\mathrm{df}$ & Sig. (2-tailed) & $\begin{array}{c}\text { Mean } \\
\text { Difference }\end{array}$ & $\begin{array}{c}\text { 95\% Confidence Interval } \\
\text { of the Difference }\end{array}$ \\
\cline { 5 - 8 } & & & & & Lower & Upper \\
\hline The explanation of the prototype is helpful & 7.424 & 31 & 0.001 & 1.000 & 0.73 & 1.27 \\
\hline
\end{tabular}

participants had significantly higher trust level when they assess the trustworthiness of Web information based on the given supportive information $(\mathrm{M}=5.21, \mathrm{SE}=0.069)$ than to assess the trustworthiness of Web information based on only the basic information provided $(\mathrm{M}=4.59, \mathrm{SE}=0.092), \mathrm{t}(63)$ $=-7.39, \mathrm{p}<0.05$. These results suggested that the trust level of the trustworthiness of Web information of the users increased if they obtained useful supportive information about that Web information. Specifically, our results suggested that when users evaluated the trustworthiness of Web information using our tool which also provides supportive information along with the Web information, the mean of their trust level increased by 0.62 percentage points as shown in Table I

\section{$B$. The users' satisfaction with the provided supportive infor- mation insofar as it helps them to evaluate the trustworthiness of Web information}

The participants were asked to rate their level of satisfaction with the supportive information and its effect on their evaluation process. We investigated that the participants think the supportive information is a good indicator to identify the trustworthiness of Web information. We used a one-sample t-test in order to investigate whether the participants were satisfied with the supportive information provided by the prototype. In this analysis, the scale used in this analysis has five points. They are not at all satisfied (1), slightly satisfied
(2), moderately satisfied (3), very satisfied (4), and extremely satisfied (5). Results from the t-test data analysis showed that the mean satisfaction level of the participants $(\mathrm{M}=3.69, \mathrm{SD}$ $=0.69)$ was statistically significantly larger than the not at all satisfied; $\mathrm{t}(31)=21.95, \mathrm{p}<0.05$ as shown in Table II. These results suggested that, on average, the participants were moderately satisfied $(M=3.69)$ with the supportive information provided.

C. The influential of the provided supportive information of prototype on the participants' judgment of the trustworthiness of Web information

We investigated that the participants think the supportive information helps to support their judgment. A one-sample t-test was conducted to compare the influence level of the supportive information provided from the prototype effects on the participants' judgment of the trustworthiness of Web information. Similar to before, the scale used in this analysis consists of five points: not at all influential (1), slightly influential (2), somewhat influential (3), very influential (4), and extremely influential (5). Results from the t-test data analysis showed that the mean of the influence on the participants (M $=3.72, \mathrm{SD}=0.68)$ was statistically significantly larger than not at all influential; $\mathrm{t}(31)=22.51, \mathrm{p}<0.05$ as shown in Table III. These results suggested that on average the participants were influenced by the supportive information provided (with 
the mean influence being $\mathrm{M}=3.72$, or somewhat influential).

\section{The explanation for why the piece of information should be trusted provided by the prototype is helpful}

In the questionnaire, the participants were asked to state their opinion regarding the perceived level of helpfulness of the explanation provided by the prototype. A one-sample ttest was conducted to compare the level of agreement of the participants over the helpfulness of the explanation on why the piece of information should be trusted by the prototype. In this analysis, a constant value, set as 3 , represents the neutral opinions (neither agree nor disagree). The score 5 represents strongly agree and conversely, the score 1 represents strongly disagree. Results from the t-test data analysis showed that the mean of the participants' agreement over the helpfulness of the explanation $(\mathrm{M}=4.00, \mathrm{SD}=0.76)$ was statistically significantly larger than the neither agree nor disagree; $\mathrm{t}(31)=7.42, \mathrm{p}<$ 0.05 as shown in Table IV. Therefore, the results suggested that, on average, the participants significantly agree that the explanation for why the piece of Web information should be trusted was helpful. In summary, the results showed that the participants experienced an increase in their trust level of Web information. In addition, the participants were satisfied with the supportive information provided to support their evaluation of the trustworthiness of Web information and they significantly agreed that the explanation provided by the prototype were helpful to support their evaluation of the trustworthiness of Web information.

\section{CONCLUSION}

In this paper, we presented a prototype which can help users to evaluate the trustworthiness of Web information. The prototype gathered the useful meta- data based on the practical trustworthiness criteria and integrated collected metadata using Linked data in order to create supportive information present to the users. We evaluated our prototype with an empirical study and the results from the study suggested that the users increased their trust level by 0.62 percentage points when they obtained useful supportive information. In addition, overall, users were satisfied with the supportive information provided to support their evaluation of the trustworthiness of Web information and significantly agreed that the additional information about the explanation provided by the prototype were helpful to support their evaluation of the trustworthiness of Web information. The work presented in this paper has proven that the prototype can help Web users to evaluate the trustworthiness of Web information. However, there are still some challenging issues that can adopt to extend our prototype in the future. One suggested challenging might be expanding the prototype in to a macro scale. Another challenging could be combining the provided criteria in prototype with the concept of privacy accountability which looking at the data that is used by the people users trust in order to provide supportive information in several perspective.

\section{REFERENCES}

[1] S. Y. Rieh and N. Belkin, "Understanding judgment of information quality and cognitive authority in the WWW," in Proceedings of the 61 st annual meeting of the american society for information science, vol. 35. Citeseer, 1998, pp. 279-289.
[2] B. Fogg, J. Marshall, T. Kameda, J. Solomon, A. Rangnekar, J. Boyd, and B. Brown, "Web credibility research: a method for online experiments and early study results," CHI'O1 extended abstracts on Human factors in computing systems, pp. 295-296, 2001.

[3] N. Wathen and J. Burkell, "Believe it or not: Factors influencing credibility on the Web," Journal of the American Society for Information Science and Technology, vol. 53, no. 2, pp. 134-144, 2002.

[4] M. A. Tate, "Information Quality Criteria for Web Resources," in Web Wisdom: How To Evaluate and Create Information Quality on the Web, 2nd ed. CRC Press, 2010, ch. 2, pp. 7-14.

[5] T. Heath and C. Bizer, "Linked data: Evolving the web into a global data space," in Synthesis Lectures on the Semantic Web: Theory and Technology, 1st ed., J. Hendler and F. Van Harmelen, Eds. Morgan \& Claypool publishers, 2011, pp. 1-136.

[6] T. Berners-Lee, "Design issues: Linked data," 2006. [Online]. Available: http://www.w3.org/DesignIssues/LinkedData.html

[7] R. S. Taylor, Value-added processes in information systems. Norwood, NJ: Ablex Publishing Corporation, 1986.

[8] S. Y. Rieh and N. Belkin, "Interaction on the Web: Scholars' Judgement of Information Quality and Cognitive Authority," Proceedings of the annual meeting-american society for information science, vol. 37, pp. $25-38,2000$.

[9] F. Naumann, "Quality-driven query answering for integrated information systems," Database Management \& Information Retrieval, vol. 2261, pp. 3-10, 29-50, 2002.

[10] C. Bizer and R. Cyganiak, "Quality-driven information filtering using the WIQA policy framework," Web Semantics: Science, Services and Agents on the World Wide Web, vol. 7, no. 1, pp. 1-10, Jan. 2009.

[11] R. Sumalatha, S. Paulraj, S. Joseph, and V. Ramaraj, "Enhanced Trustworthy and High-Quality Information Retrieval System for Web Search Engines," Internation Journal of Computer Science, vol. 5, pp. $38-42,2009$.

[12] B. J. Fogg, C. Soohoo, D. Danielson, L. Marable, J. Stanford, and E. R. Tauber, "How do people evaluate a Web site's credibility? Results from a large study," Tech. Rep., 2002.

[13] H. Miyamori, S. Akamine, Y. Kato, K. Kaneiwa, K. Sumi, K. Inui, and S. Kurohashi, "Evaluation data and prototype system WISDOM for information credibility analysis," Internet Research, vol. 18, no. 2, pp. 155-164, 2008.

[14] P. K. Atrey, H. Ibrahim, M. Anwar Hossain, S. Ramanna, and A. El Saddik, "Determining trust in media-rich websites using semantic similarity," Multimedia Tools and Applications, vol. 60, no. 1, pp. 6996, Apr. 2011.

[15] J. Pattanaphanchai, "Trustworthiness of Web Information Evaluation Framework," Doctoral Thesis, University of Southampton, 2014.

[16] Ecma International, "ECMA-404 The JSON Data Interchange Format," Tech. Rep. October, 2013. [Online]. Available: http://www.ecmainternational.org/publications/files/ECMA-ST/ECMA-404.pdf

[17] Eprints, "Eprints," 2011. [Online]. Available: http://www.eprints.org/

[18] J. Pattanaphanchai, K. O'Hara, and W. Hall, "Trustworthiness criteria for supporting users to assess the credibility of web information," in the 22nd international conference on World Wide Web companion, 2013, pp. 1123-1130.

[19] C. Bizer, R. Cyganiak, T. Gauss, and O. Maresch, "The TriQL. P browser: Filtering information using context-, content-and rating-based trust policies," in Proceedings of the Semantic Web and Policy Workshop at the 4th International Semantic Web Conference, vol. 7, 2005, pp. 1220.

[20] J. E. Hirsch, "An index to quantify an individual's scientific research output," Proceedings of the National Academy of Sciences of the United States of America, vol. 102, no. 46, pp. 16569-16 572, 2005.

[21] J. Carroll, C. Bizer, P. Hayes, and P. Stickler, "Named graphs, provenance and trust," in Proceedings of the 14th international conference on World Wide Web. New York, New York, USA: ACM Press, 2005, pp. 613-622.

[22] C. Bizer, "Semantic Web Publishing Vocabulary (SWP) User Manual," Tech. Rep. November, 2006.

[23] J. J. Carroll and P. Stickler, "Trix: RDF Triples in xml," 2007. [Online]. Available: http://www.citeulike.org/group/1142/article/1160232 